\title{
The neo-Luddite's lament: excessive upgrades in the software industry
}

\author{
Glenn Ellison* \\ and \\ Drew Fudenberg**
}

We examine two reasons why a monopoly supplier of software may introduce more upgrades than is socially optimal when the upgrade is backward but not forward compatible, so users who upgrade reduce others' network benefits. One explanation involves a commitment problem: profits and social welfare may suffer because ex post the monopolist will want to sell the upgraded product to new consumers. The second involves consumer heterogeneity. Here oversupply arises from the difference between the externality that upgrades impose on the marginal and average consumer, and from the effect of upgrades on sales of the base good.

\section{Introduction}

- This article explores a monopolist's incentives to provide upgraded versions of its software, focusing on how market power, commitment problems, and price discrimination may lead a monopolistic supplier of a network good to introduce upgrades when social welfare would be higher without them.

Our primary motivation is the markets for software for personal computers. The development of the Internet and of new operating systems has spurred a desire for more features, and increases in computer power have made improvements feasible. Perhaps as a result, in the last decade, most popular packages have introduced upgrade after upgrade. Recently, however, it has become increasingly common to hear frustrated consumers decrying this "progress." In particular, users complain that in the Internet era, sales of new "backward-compatible" upgrades are an exploitation of network externalities designed to "force" people to buy upgrades they don't want (and that are also inefficient because they leave others struggling with incompatibility).

From the very beginning, upgrades have brought some complaints. For example, although Lotus 1-2-3 release 2 (September 1985) was hailed for its ability to access EMS memory and use a math coprocessor, it was criticized as a memory hog (it

* Massachusetts Institute of Technology; gellison@mit.edu.

** Harvard University; dfudenberg @ harvard.edu.

We thank Jae Nahm, Patrick Rey, Jean Tirole, and two referees for helpful comments, and Peter Eso for outstanding research assistance. The National Science Foundation provided research support via grant nos. SBR-9818534 and SBR-9730181. The first author was also supported by a Sloan Research Fellowship and by a fellowship from the Center for Advanced Study in the Behavioral Sciences at Stanford University. 
occupied $180 \mathrm{~K}$ of RAM versus $100 \mathrm{~K}$ for $1-2-3$ release $1 \mathrm{~A}$ ) and for its lack of file compatibility with earlier versions of 1-2-3. To incorporate new features (and to conserve on disk space by no longer storing large columns of blank cells) the developers of release 2 had chosen to use a new file format (.WK1). While release 2 could read some worksheets stored in the old format (.WKS), this was only true of some worksheets, and release 2 could not write files in the old format. This lack of "backward compatibility" caused many firms to hold back on upgrading. ${ }^{1}$

Lotus learned from the release 2 experience. Although subsequent releases of 1-2-3 included new file formats (.WK3, .WK4, and .WK5), they were fully backward compatible, meaning that they could read and write files in older formats. Potential purchasers of upgrades were no longer deterred by fears that they would be unable to use their old files or files produced by others. The evolution of most spreadsheet, word processing, database, and statistical software has followed a similar pattern. For example, Excel, Word, WordPerfect, dBase, and Stata have all introduced backwardcompatible upgrades with new file formats at least three times in the past decade. ${ }^{2}$

While backward compatibility is now standard, in the last few years there has been a new wave of complaints about upgrades. The new complaints center on what users believe are "forced upgrades" - upgrades that are costly to buy, learn, and install and that provide little benefit, but which consumers feel they must buy in order to maintain compatibility with the rest of the world. ${ }^{3}$ One might hope that companies would not intentionally introduce upgrades that are a nuisance, since doing so would lead forwardlooking consumers to pay less when initially buying the product, so that inefficient upgrades would make the company worse off. Unsurprisingly, though, there are a number of reasons why this turns out not to be the case.

We analyze two closely related models, both of which make strong assumptions about production technology, consumer demand, and market institutions. On the production side, we assume that software can be produced at zero marginal cost, but that consumers incur setup costs with each successive version of the product. We also suppose that new versions of the software are backward compatible with past versions, but that old versions are not forward compatible with the new ones. We suppose that the consumers' utility from the software stems partially from network externalities, so that consumers prefer to use software that is compatible with as many other consumers as possible, and that because new software is backward compatible, users of the newest

${ }^{1}$ See "1-2-3, Release 2 Is Not a Must Buy," Infoworld, December 2, 1985, "Compatibility Problems Block Upgrade Path for 1-2-3 Users," Infoworld, December 9, 1985, and "Lotus Upgrade's Compatibility Lessens Users' Release 2 Anger; Release 2.01 of 1-2-3 Targets 'Critical Issues'," Computerworld, August 4, 1986. Other sources claim the incompatibilities eventually worked to Lotus's advantage, as consumers who feared being "out of step with the crowd" bought the upgrade ("Sales of 1-2-3 Upgrade Are Sparked by Users' Fear of Incompatibility," PC Week, May 13, 1986).

${ }^{2}$ Backward compatibility, of course, has not solved all the problems of software upgrades. For example, it is still common to hear wishes that new versions of software had been designed to use less disk space or omitted unwanted features, e.g., the Microsoft Word "paper clip guy" (who is finally being made removable in Office 2000!).

${ }^{3}$ Zeid Nasser, for example, writes "It is like forcing us all to upgrade. ... If you exchange files with friends or colleagues, then one of them is bound to be a user of a newer version of Word. So you will have to follow suit to read the files sent to you. It really makes you wonder, if we are playing according to a grand plan, set for us in Redmond, Washington - the new headquarters of the modern computer-run world ... Although this may sound strange, coming from an information technology writer ... Surely, tens of thousands of users worldwide feel the same about the 'forced upgrade' policy that we are being subjected to." (" "Forced Upgrading' in the World of Word," The Star, August 20, 1998.) Likewise, Paul Rubens writes "While many organizations ... appear unconvinced about the suite's advantages over existing products, they seem resigned to the fact that they will ultimately have no choice but to use Office 97." ("Office 97-To Buy or Not To Buy?" The Dominion, November 3, 1997.)

(C) RAND 2000. 
version enjoy the largest network benefits. ${ }^{4}$ On the institutional side, we assume that the monopolist is able to offer upgrade discounts (but not penalties!) to consumers who have purchased previous versions of the good; this means that our article corresponds to what Fudenberg and Tirole (1998) call the "semi-anonymous" case. We also assume that there is no rental market; this is descriptively realistic, and it avoids introducing the commitment problem that would come from the monopolist exploiting consumers who have sunk their setup costs. Although the model is too simple in many respects to be an accurate description of any particular software market (for example, there are no competitors and only two periods), we were led to our assumptions by our experience with word processing software, and that market may be useful to keep in mind when evaluating our assumptions.

It is already known that in competitive markets with network externalities there can be equilibria in which consumers are too quick to upgrade to new products, but in simple models these equilibria arise solely as a coordination failure, because there is typically another equilibrium with efficient upgrade decisions. We identify two additional forces that lead to excessive introduction of upgrades even when consumers coordinate their decisions.

First of all, we analyze a dynamic model with identical consumers to show that when there is a steady inflow of new customers to the market, the monopolist may suffer from a commitment problem: ex post it may prefer to sell an upgraded, more highly valued version to the new customers, but this forces the old customers to either incur the costs of learning how to use the new version or suffer a lack of compatibility with the new customers. Since the resulting loss in first-period profits may be larger than the second-period gain, the monopolist and society as a whole may both be worse off than if the monopolist could commit itself not to offer the upgrade. The implications of this commitment problem can vary with the resolution of the second-period coordination game between consumers who already own the old version.

If these consumers coordinate on the equilibrium that is Pareto optimal for them, then the monopoly outcome is efficient whenever the social optimum is either upgrading or incompatible networks, and inefficient outcomes occur only when a monopolist who had commitment power would not introduce the new good. Here inefficiency occurs because the monopolist without commitment introduces the new good, which leads either to inefficient upgrading by old consumers or to inefficient incompatibility between old and new consumers. If instead the consumers coordinate on the equilibrium that the monopolist prefers, an additional form of inefficiency is possible - the monopoly outcome can be upgrades when the social optimum is incompatible networks.

The monopolist's commitment problem here is similar to that in Waldman (1993), where a monopoly without commitment power lowers both profits and welfare by selling a new incompatible version of its product. Our model differs in a couple of ways. First, in our model the upgrade is backward compatible, which seems a better description of some of the software examples. Second, the upgrade cost is borne by consumers, so that the commitment problem cannot be resolved with a promise to provide free upgrades. Our analysis also differs in that we analyze the implications of two possible resolutions of the coordination problem faced by new consumers. Choi

${ }^{4}$ In our model, the owner of the newer software suffers no loss at all from the fact that the old software is not forward compatible. This is an extreme assumption. Both parties typically suffer when one person sends another a file that cannot be read, but our impression is that the recipient loses more. (An example where only the recipient loses is when a referee sends a report in a format an editor cannot read: the referee is happy, since he has fulfilled his obligation to send his report on time, and the editor is unable to make a decision until the hard copy arrives.)

(C) RAND 2000 
(1994) also analyzes the commitment problem of a durable-good monopolist with network externalities, showing most notably that a monopolist may choose to make a new product incompatible even though perfect compatibility would entail no additional cost. The social optimum in Choi's model is always to introduce the new product and make it compatible, so that (in contrast to our model) it is never the case that upgrades are introduced too often. The commitment problem in Choi is also somewhat different, in that it is purely a matter of the monopolist being tempted to try to extract more surplus from old customers. ${ }^{5}$

Our second model examines a population of heterogeneous consumers in order to discuss price discrimination motivations for excessive upgrading that may be present even if the monopolist is able to commit to an upgrade policy. Because the commitment assumption makes the dynamics uninteresting, we simplify here by analyzing a static model, in which the monopolist sells the basic model of the good and also sells an upgraded version. This lets us focus on the key issue, which is the way the heterogeneity of preferences relates to the network effects. It seems most natural to us that consumers who have a higher "inherent" value for a product will also place a greater value on network benefits, but all past work on network effects that we know of has used an "additive" specification in which a consumer's network benefit is independent of his valuation for the base good. This distinction is important in our setting because when a monopolist sells a backward-compatible upgrade, it is imposing a negative externality on users who don't upgrade. Because a monopolist's revenue depends only on the surplus of the marginal buyer, it may have an incentive to introduce too many upgrades if the negative externality is less strong for the marginal buyer than for the average one. In contrast, with the additive specification, the motivation mentioned above is not present, so for a fixed level of sales of the base good the monopolist will always sell too few upgrades for the standard monopoly reason.

This, however, is not the whole story. Because of the negative externality, the sales level of the base good is increased when the upgrade is not available. This raises the possibility that social welfare could be improved by a ban on upgrades, and it turns out that this is sometimes the case. Specifically, even with the additive specification, welfare is increased by banning the upgrade whenever unconstrained sales of the upgrade are sufficiently small.

In addition to the articles mentioned earlier, this article is related to many lines of research. The discussion of commitment problems in durable-goods monopoly dates back to Coase (1972), with key contributions by Bulow (1982) and Stokey (1981). More recently, Fudenberg and Tirole (1998) study the monopoly pricing of successive generations of a durable good in several different institutional contexts, including the "semi-anonymous" case that we study here. There is also a large literature on competition in networks, including Farrell and Saloner (1985), Katz and Shapiro (1986), Nahm (1999), and Bensaid and Lesne (1996). ${ }^{6}$ Finally, our model of excess upgrades under commitment is related to work on how the desire to price discriminate influences the provision of quality and product diversity, notably Spence $(1975,1976)$, Mussa and Rosen (1978), and Deneckere and McAfee (1996), although none of these articles considered cases where the consumption of some consumers imposed an externality on

\footnotetext{
${ }^{5}$ Choi also analyzes the case where the monopolist is unable to price discriminate between owners of the old good and new consumers. Related but less similar commitment problems arise in models of durablegoods monopolists with second-hand markets but without network externalities, as in the "planned obsolescence" articles of Waldman (1996) and Nahm (1998).

${ }^{6}$ This article claims to model durable goods, but the equations used don't seem to be consistent with that interpretation.

(1) RAND 2000
} 
others; in a sense the upgrades here are not "damaged goods" but "damaging" ones, like cigar smokers in a restaurant, which some restaurants nonetheless encourage.

\section{Identical consumers}

- In this section we suppose that all consumers are identical, so that the monopolist will be able to extract the full social surplus from whatever goods it produces. Besides simplifying the analysis, this assumption also ensures that any deviations of the monopolist's output path from social efficiency cannot be attributed to price discrimination.

We consider a two-period model, with periods $t=1$ and $t=2$. In the first period, the monopolist can produce the first generation of the software, which has "base value" $q_{1}$. In period 2 the monopolist has the option of introducing a new version, with base value $q_{2}$; we let $q_{\Delta}=q_{2}-q_{1}$. For simplicity we assume that both producing existing goods and developing new ones is costless; allowing for fixed costs of developing each generation would make upgrades less frequent but would have only the obvious impact on our conclusions.

Each consumer incurs cost $c$ the first time he starts to use the software, and an additional cost $c_{u}<c$ when learning to use a new version. Also, each consumer receives network benefit $a x$ from using the good in a period when the mass of consumers using a compatible program is $x$. To model the effects of backward compatibility, we assume that consumers of the old software derive network benefits only from other consumers of old software, while consumers of new software get the full network benefit from both groups of consumers. ${ }^{7}$ We suppose that utility is linear in income, and that consumers and the firm have a common discount factor $\delta$; we also suppose that $(1+\delta) q_{1}>c$, so that even without network benefits it would be more efficient for first-period consumers to adopt version 1 than for them never to adopt the good at all. We also suppose that there is an inflow of customers into the market each period, with mass $\lambda_{1}$ arriving in period 1 and $\lambda_{2}$ arriving in period 2; to normalize we set $\lambda_{1}+\lambda_{2}=1$. To cut down on the number of cases under consideration we also assume that $q_{1}+\alpha \lambda_{1}>(1-\delta) c+\delta c_{u}$, so that it is optimal for first-period consumers to consume in both periods as opposed to waiting until period 2 .

Since utility is linear in money, we can measure total welfare in the economy by summing over agents. From this perspective it makes sense to ask whether the market provides new versions when it would be more efficient not to do so, but note that because the new version is better and is backward compatible, at constant prices the new consumers are always better off with the new version. We compute that total welfare is

$$
\begin{array}{ll}
W_{N}=\lambda_{1}\left[(1+\delta) q_{1}+\alpha \lambda_{1}+\delta \alpha-c\right]+\lambda_{2} \delta\left[q_{1}+\alpha-c\right] & \text { if good } 2 \text { is not used; } \\
W_{U}=\lambda_{1}\left[q_{1}+\delta q_{2}+\alpha \lambda_{1}+\delta \alpha-c-\delta c_{u}\right]+\lambda_{2} \delta\left[q_{2}+\alpha-c\right] & \text { under the "upgrade policy," }
\end{array}
$$

where good 2 is introduced in period 2 and all old consumers upgrade; and

$$
\begin{array}{r}
W_{I}=\lambda_{1}\left[(1+\delta) q_{1}+(1+\delta) \alpha \lambda_{1}-c\right]+\lambda_{2} \delta\left[q_{2}+\alpha-c\right] \\
\text { with "incompatible networks,", }
\end{array}
$$

where good 2 is introduced yet the old consumers stick with the incompatible good 1 .

${ }^{7}$ If we let $x_{1}$ be the size of the old network and $x_{2}$ the size of the new one, then the network benefits provided by the two goods are $\alpha x_{1}$ and $\alpha\left(x_{1}+x_{2}\right)$ respectively. A richer model would have four parameters $\alpha_{i j}$, with the network benefit of good $i$ equal to $\alpha_{i i} x_{i}+\alpha_{i j} x_{j}$.

() RAND 2000 
(Because the new version is backward compatible, only the old customers lose network benefits from incompatibility.)

Comparing the first two expressions shows that $W_{N}>W_{U}$ when $\lambda_{1} c_{u}>q_{2}-q_{1}=q_{\Delta}$, so that the upgrade cost to old users exceeds the gain in all users' second-period utility. Similarly, $W_{N}>W_{I}$ when $\alpha \lambda_{1} \lambda_{2}>\lambda_{2} q_{\Delta}$ or $\alpha \lambda_{1}>q_{\Delta}$, so the loss from incompatibility is greater than the utility benefit to new users from the better good, and $W_{I}>W_{U}$ when $\lambda_{1} q_{\Delta}<\lambda_{1} c_{u}-\alpha \lambda_{1} \lambda_{2}$ or $q_{\Delta}+\alpha \lambda_{2}<c_{u}$, so the cost of upgrade is greater than the sum of the quality improvement and the gains from a larger network.

To determine when each regime is optimal, we combine these conditions, yielding the following result.

Proposition 1. (i) If $\alpha>c_{u}$, so that network effects are large compared to upgrade costs, the optimum is that old consumers upgrade when $q_{\Delta}>c_{u} \lambda_{1}$, and do not use good 2 when the inequality goes the other way.

(ii) If $\alpha<c_{u}$, so that network effects are comparatively small, upgrading is optimal when $q_{\Delta}>c_{u}-\alpha \lambda_{2}$, incompatibility is optimal when $\lambda_{1} \alpha<q_{\Delta}<c_{u}-\alpha \lambda_{2}$, and policy $N$ is optimal when $q_{\Delta}<\alpha \lambda_{1}$. Each of these three regions is nonempty.

Combining parts (i) and (ii) yields the characterization of when each regime is optimal that is displayed in Figure 1, which displays the pairs $\left(\alpha, q_{\Delta}\right)$ for which each of the three policies $N, I$, and $U$ is optimal. As the figure shows, the socially optimal policy is

(a) Upgrade if $\alpha>c_{u}$ and $q_{\Delta}>c_{u} \lambda_{1}$, or if $\alpha<c_{u}$ and $q_{\Delta}>c_{u}-\alpha\left(1-\lambda_{1}\right)$.

(b) Use incompatible goods if $\alpha<c_{u}$ and $\lambda_{1} \alpha<q_{\Delta}<c_{u}-\alpha\left(1-\lambda_{1}\right)$.

(c) Use only good 1 if $\alpha>c_{u}$ and $q_{\Delta}<c_{u} \lambda_{1}$ or if $\alpha<c_{u}$ and $q_{\Delta}<\alpha \lambda_{1}$.

We now turn to the examination of the monopolist's policy when it lacks commitment power and can only offer short-run sales contracts. We suppose that the monopolist can offer an upgrade discount to owners of the old version, so that if the new version is introduced, then the monopolist chooses one price $p_{2}$ to charge new consumers and a second one $p_{u} \leq p_{2}$ for the upgrade. ${ }^{8}$ New consumers prefer version 2 of the good, and it is costless to provide it. Subject to the caveat below, this implies that once the second period arrives, the monopolist will never choose to withhold the new version from the market. Since withholding the good is sometimes socially optimal, it is already clear that the outcome may have more progress than is efficient; to obtain more specific results we now characterize the equilibrium outcomes.

First, we note that as is usual in studies of network goods, consumers' purchasing decisions given announced prices resemble a coordination game and can have multiple equilibria. The standard assumption is that buyers with the same preferences act as though they were a single player, which is equivalent to selecting the continuation equilibrium that is best for the buyers. ${ }^{9}$ For the new buyers, this coordination rule implies that they will purchase good 2 if and only if

$$
q_{2}+\alpha-c-p_{2} \geq \max \left\{0, q_{1},+\alpha-c-p_{1}\right\}
$$

\footnotetext{
${ }^{8}$ Since all consumers are the same, the monopolist would never wish to set $p_{u}>p_{2}$. When we consider heterogeneous consumers in Section 3 this need no longer be true, for customers who purchased in the first period have relatively high valuation for the good, and so their utility from an upgrade may be greater than the profit-maximizing price to a new customer.

${ }^{9}$ Note that choosing the best continuation equilibrium is not the same as choosing the continuation that maximizes the buyers' overall equilibrium payoff, since a "tougher" purchasing rule may lead to lower prices.

(c) RAND 2000
} 
FIGURE 1

THE SOCIALLY OPTIMAL OUTCOME

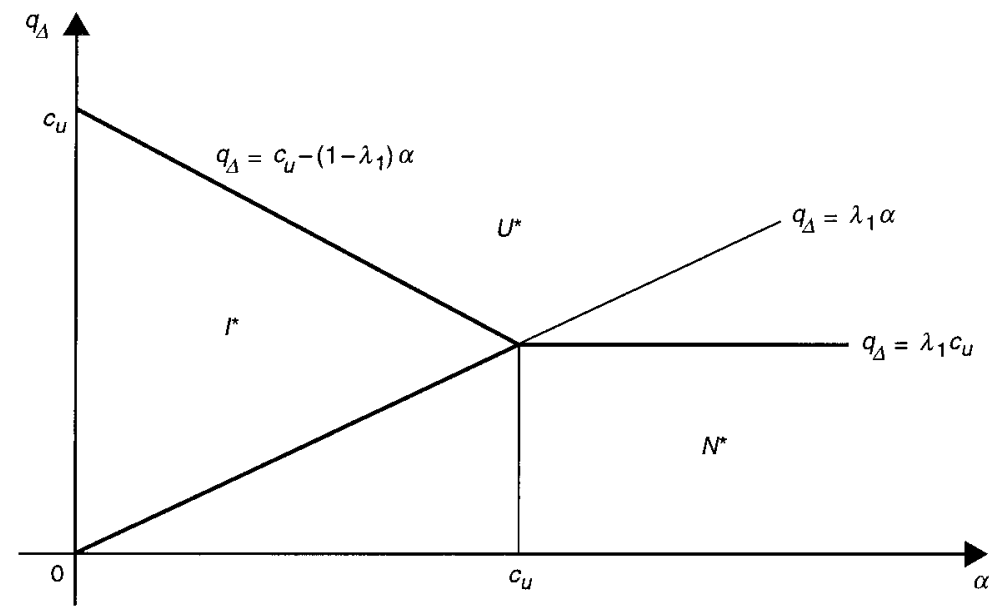

and otherwise purchase good 1 if $q_{1}+\alpha-c-p_{1} \geq 0$. This is the best continuation equilibrium for them given the announced price. However, it is also an equilibrium for the new consumers to use other rules, such as "purchase good 2 if and only if $q_{2}+\alpha \lambda_{1}-c-p_{2} \geq \max \left\{0, q_{1}+\alpha \lambda_{1}-c-p_{1}\right\}$ " and "purchase good 2 if and only if $q_{2}+\alpha \lambda_{1}-c-p_{2} \geq \max \left\{0, q_{1}+\alpha-c-p_{1}\right\}$." ${ }^{10}$ We will follow convention here and suppose that new buyers coordinate on the equilibrium that is best for them. Given this equilibrium selection, the fact that the new good is backward compatible with the old one implies that the monopolist makes more profits from the new consumers by selling good 2 at price $p_{2}=q_{2}+\alpha-c$ than it could make selling good 1 .

We will explore the consequences of two possible coordination rules for old consumers in their upgrade decision, because alternate and equally "unbiased" rules can have what we think are interesting efficiency consequences, and we do not have a strong belief about which rule is more descriptive. Note that the second-period payoff to upgrading is $q_{2}+\alpha-c_{u}-p_{u}$, irrespective of the play of other old customers, while the second-period payoff to continued use of good 1 is $q_{1}+\alpha \lambda_{1} x_{1}+\alpha \lambda_{2} x_{2}$, where $x_{1}$ is the share of old customers who continue to use good 1 and $x_{2}$ is the share of new consumers who purchase good 1. Hence in the subgame corresponding to upgrade prices $p_{u}$ with $p_{u}<q_{\Delta}+\alpha \lambda_{2}\left(1-x_{2}\right)-c_{u} \equiv p_{u}^{*}$, the unique equilibrium response is to purchase, since this is the best response regardless of the play of other consumers; for $p_{u}>q_{\Delta}+\alpha \lambda_{1}+\alpha \lambda_{2}\left(1-x_{2}\right)-c_{u} \equiv \bar{p}_{u}$, the unique equilibrium response is to not upgrade; and in the subgame following a price $p_{u}^{*} \leq p_{u} \leq \bar{p}_{u}$, there are two purestrategy equilibria_- "all upgrade" and "all stick with good 1" — as well as an equilibrium in mixed strategies. At any positive price in this region the firm's payoff in this subgame is highest at the equilibrium where everyone upgrades; conversely, at any price in this region the customers' payoff in the subgame is higher if they all stick with good 1. We will investigate the two "uniform" selection rules, the "reluctant"

${ }^{10}$ The latter rule can induce the monopolist to sell good 1 in the second period instead of good 2. There can also be equilibria in which the choice of coordination rule depends on the first-period price. These contingent rules can be used to support a range of first-period and upgrade prices as equilibria. 
rule, "upgrade when $p_{u} \leq p_{u}^{*}$," and the "eager" rule, "upgrade when $p_{u} \leq \bar{p}_{u}$." With either rule, the monopolist's profits in the upgrade market are decreasing in $x_{2}$. Hence, we can assume without further loss of generality that the monopolist does not sell good 1 in period 2 and simplify the formulas above to $p_{u}^{*}=q_{\Delta}+\alpha \lambda_{2}-c_{u}$ and $\bar{p}_{u}=q_{\Delta}+\alpha-c_{u}{ }^{11}$

If buyers coordinate on the reluctant equilibrium, the monopolist will set $p_{u}=\max \left\{0, p_{u}^{*}\right\}$. The social payoff to upgrading is precisely the welfare gained by the customers who upgrade minus their setup cost, which equals $p_{u}^{*}$, so the upgrade decision maximizes total welfare, and the monopolist's profit on the upgrade market equals the social surplus there. In contrast, if buyers use the "eager" rule and upgrade whenever $p_{u} \leq \bar{p}_{u}$, the monopolist sets $p_{u}=\bar{p}_{u}$, and if $p_{u}^{*}=q_{\Delta}+\alpha \lambda_{2}-c_{u}<0<\bar{p}_{u}$, the result is that old buyers upgrade when it would be more efficient for them to stick with the old version.

Working back to the first period, we note first that the facts that $p_{2}$ extracts all the surplus from new buyers and that old and new buyers are identical imply that old buyers receive net payoff of zero from waiting to purchase in period 2, so they are willing to pay any price $p_{1}$ that is less than the expected discounted stream of benefits from purchasing. Of course, these benefits depend on the buyers' expectations about second-period play: If they expect that everyone will use the old good in the second period, they also expect that the second-period price for the old good will extract all of the second-period surplus, so they are willing to pay $(1+\delta) q_{1}+\alpha \lambda_{1}+\delta \alpha-c$ to purchase good 1 in period 1 . If they expect the incompatible regime, they are willing to pay any first-period price below $(1+\delta) q_{1}+(1+\delta) \alpha \lambda_{1}-c$, and if they expect that they will have to upgrade, they will pay up to $q_{1}+\delta q_{2}+\alpha \lambda_{1}+\delta \alpha-c-\delta c_{u}-\delta p_{u}$. Note that in the upgrade case the total discounted payments by the old consumers are independent of the upgrade price $p_{u}$, as higher upgrade prices are offset by lower firstperiod prices, and that in all three cases the total discounted payments extract all of the consumer surplus. This means that the monopolist's present value would be maximized by the efficient allocation rule. Moreover, in cases where

$$
q_{\Delta}+\alpha \lambda_{2}-c_{u}<0<p_{u}<\bar{p}_{u}
$$

and the old consumers upgrade, the monopolist has a lower present value than in the equilibrium where old consumers are "tougher" and reject all $p_{u}>p_{u}^{*}$, even though this equilibrium gives the monopolist higher profit in the second period.

Combining these observations with the characterization of the efficient policy leads to the following results.

Proposition 2. Suppose that old consumers use the reluctant upgrade rule. Then the monopoly outcome is for old consumers to upgrade if and only if $q_{\Delta}+\alpha \lambda_{2}-c_{u}>0$. As illustrated in Figure 2, this is the same as the efficient outcome described in Proposition 1 except in two regions of parameter space:

${ }^{11}$ The monopolist could never gain by making the good incompatible rather than backward compatible if backward compatibility is free and the choice is made in the second period. To see this, note first that incompatibility never increases the amount new consumers are willing to pay for the new good at any fixed sizes of the two networks. Thus, if the old consumers do not upgrade under compatibility, then incompatibility reduces the monopolist's profit. If old consumers do upgrade under compatibility, the price they pay depends on their coordination rule. The "eager" price (the value of the upgrade if everyone upgrades) is not affected by the compatibility decision, so in this case the monopolist is just indifferent between incompatibility and backward incompatibility, while the "reluctant" price (and indeed all other possible equilibrium prices) are strictly lower under incompatibility.

(c) RAND 2000. 
FIGURE 2

THE MONOPOLIST'S REGIMES AND EFFICIENCY IN THE $\left(\alpha, q_{\Delta}\right)$ SPACE WHEN CONSUMERS USE THE "RELUCTANT" COORDINATION RULE

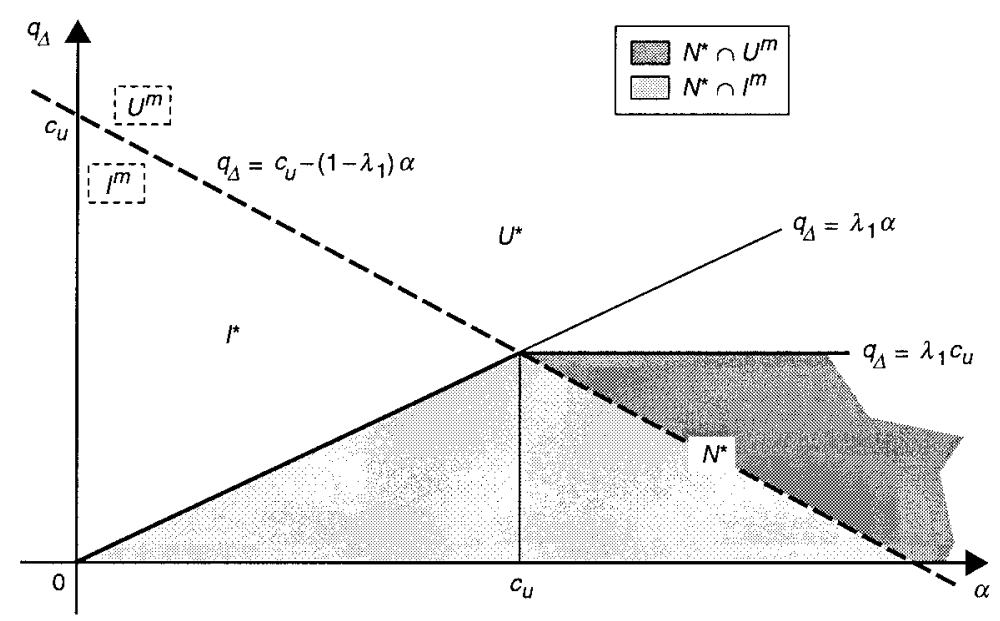

(i) If $q_{\Delta}>c_{u}-\alpha\left(1-\lambda_{1}\right)$ and $q_{\Delta}<\lambda_{1} c_{u}$, the monopoly outcome is upgrading while the social optimum is to sell only good 1 .

(ii) If $q_{\Delta}<c_{u}-\alpha\left(1-\lambda_{1}\right)$ and $q_{\Delta}<\alpha \lambda_{1}$, the monopoly outcome is incompatibility and the social optimum is to sell only $\operatorname{good} 1$.

Proposition 3. Suppose that old consumers use the eager upgrade rule. Then the monopoly outcome is for old consumers to upgrade if $q_{\Delta}+\alpha-c_{u}>0$. As illustrated in Figure 3, this is the same as the efficient outcome except

(i) If $q_{\Delta}>c_{u}-\alpha$ and $q_{\Delta}<\min \left\{\lambda_{1} c_{u}, \lambda_{1} \alpha\right\}$, the monopoly outcome is upgrading while the social optimum is to sell only good 1 .

\section{FIGURE 3}

THE MONOPOLIST'S REGIMES AND EFFICIENCY IN THE $\left(\alpha, q_{\Delta}\right)$ SPACE WHEN CONSUMERS USE THE "EAGER" COORDINATION RULE

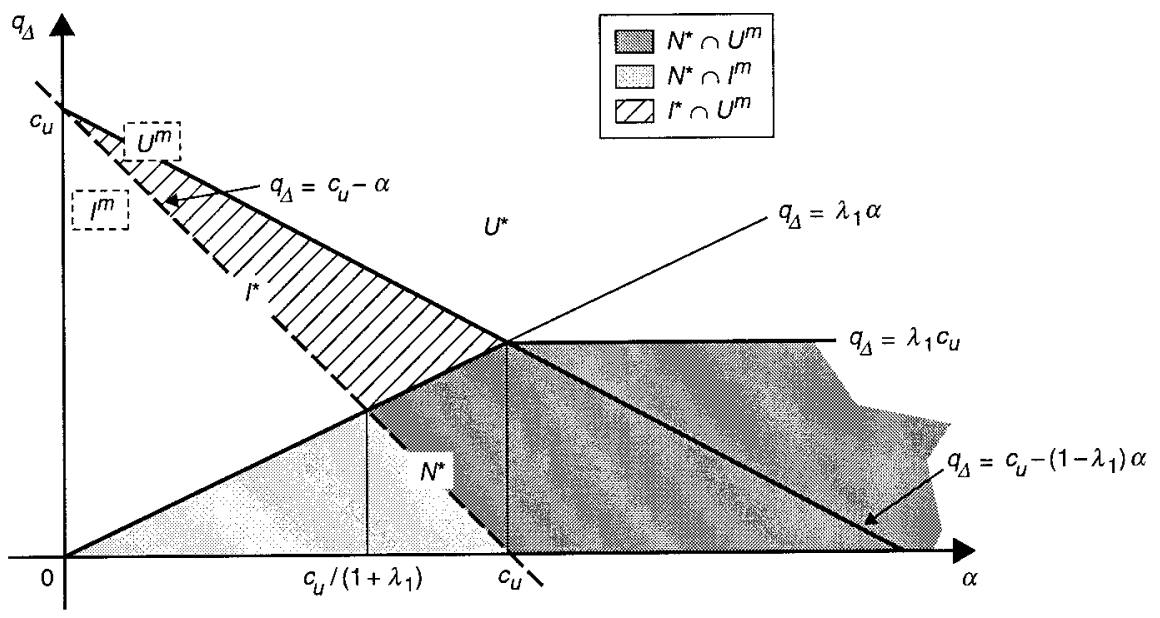


(ii) If $q_{\Delta}<c_{u}-\alpha$ and $q_{\Delta}<\lambda_{1} \alpha$, the monopoly outcome is incompatibility and the social optimum is to sell only good 1 .

(iii) If $q_{\Delta}<c_{u}-\alpha\left(1-\lambda_{1}\right), q_{\Delta}>c_{u}-\alpha$ and $q_{\Delta}>\lambda_{1} \alpha$, the monopoly outcome is upgrading while the social optimum is incompatible networks.

Comparing Figures 2 and 3 shows two differences between them. The first is the appearance of the region described in part (iii) of Proposition 3, where consumers upgrade even though incompatibility is optimal. This region does not occur with the reluctant equilibrium selection that is considered in Proposition 2 and Figure 2, because with that equilibrium selection the monopolist captures all the second-period rents given that the new good is going to be introduced, so the choice between upgrading and incompatibility is made efficiently. The second difference between the figures occurs in cases where the social optimum is to withhold good 2: In Figure 3 the region where the monopolist induces upgrades is larger, and the region where the monopolist induces incompatibility is smaller, than in Figure 2. Looking at the figures, we can also see that network externalities do not cause firms to introduce more upgrades than they would in the absence of network externalities. Rather, if there are no network externalities, then both the social optimum and the monopoly outcome are to bring any improvements to the market. As network externalities become more important it becomes socially optimal to sometimes withhold innovations, but in this model the monopolist never withholds them. ${ }^{12}$

Finally, while we have discussed the inefficient upgrading of this section as resulting from the inflow of new consumers and a lack of commitment power, the same inefficiency could arise as a reaction to potential competition. Consider an expanded version of our model where the monopolist is able to commit to whether or not he will produce an upgrade before the first period, and where a potential entrant may enter by paying a fixed development cost between the first and second periods. To deter entry the monopolist may need to develop the upgrade, and once the upgrade is developed the monopolist's pricing decision will lead to inefficient incompatibility or upgrading exactly as above.

\section{Heterogeneous consumers and commitment}

- Overview. Now we turn to the interaction of the decision of whether to offer an upgrade with the monopolist's desire to extract as much surplus as possible from consumers with heterogeneous preferences. To keep things simple, and to highlight that the issues we study here are distinct from the commitment problem studied in the previous section, we will assume that the monopolist can fully commit itself to a sequence of rental prices. Given this strong assumption, the problem is not inherently dynamic, so we further simplify by studying a static problem. We consider a monopolist that sells two goods of quality $q_{1}$ and $q_{2}, q_{1}<q_{2}$, where good 2 is "backward compatible" with good 1 but good 1 is not compatible with good 2. Because the model is static, it is immaterial whether costs of the two goods, $c_{1}$ and $c_{2}$, are viewed as production costs of the monopolist or as training costs that are identical for all consumers; we will view them as production costs here because it streamlines the exposition. In the absence of network effects $(\alpha=0)$, the model reduces to the rental problem studied

${ }^{12}$ Whenever it is optimal to withhold the new good, a monopolist who could commit to the design of the new good in period 1 might want to make the new good incompatible rather than backward compatible (or damage it in other ways) as a way of committing itself not to produce the improvement. In our earlier remark on incompatibility we assumed that such commitments were not possible.

(C) RAND 2000 
in Fudenberg and Tirole (1998), so our work can be seen as extending their analysis of multigood pricing to situations with network externalities.

We will see that the nature of the monopolist's pricing problem depends on whether the value that consumers attach to the network externality is correlated with their value for the "inherent" services of the good. Formally, we suppose that there is a continuum of consumers with types $\theta$ uniformly distributed on [0,1], and that type $\theta$ assigns utility $u=\theta q+\alpha f(x, \theta)$ to a good of quality $q$ and network size $x$. The issue is then the sign of the cross-derivative $\partial^{2} f / \partial x \partial \theta$. Previous work on compatibility issues has used the "additive specification" $u=\theta q+a x$, where $f(x, \theta)=x$ so the value of the network is the same for all consumers; we analyze this specification below. However, we think it is more plausible that someone who has a higher value for the good also assigns more importance to the size of its network. We therefore also analyze the case of "multiplicative preferences," in which type $\theta$ 's utility for a good of quality $q$ and network size $x$ is $\theta(q+\alpha x)$. In this case, the monopolist does not capture all of the benefits that the network provides to consumers, and so the monopolist can sell more of the upgrade than is optimal even given its sales of the base good. ${ }^{13}$

With either sort of preferences, any pricing scheme will segment the consumers into three (possibly empty) groups: consumers with values below some $\theta_{1}$ will not purchase, consumers in the interval $\left[\theta_{1}, \theta_{2}\right]$ will purchase good 1 , and consumers in $\left[\theta_{2}, 1\right]$ will purchase good 2 . As is standard in the mechanism design literature, we will find it easier to treat the cutoffs $\theta_{1}, \theta_{2}$ as the control variables, with the prices set by the conditions that the marginal consumers should be indifferent between their assigned allocation and their next-best choice. Moreover, as in Fudenberg and Tirole (1998), it is simplest to view the monopolist as selling good 1 to all consumer types in the interval $\left[\theta_{1}, 1\right]$ and then selling an "upgrade" at price $p_{u}=p_{2}-p_{1}$ to consumers in $\left[\theta_{2}, 1\right]$, where the price $p_{1}\left(\theta_{1}, \theta_{2}\right)=\theta_{1} q_{1}+\alpha f\left(\theta_{1}, \theta_{2}-\theta_{1}\right)$ makes consumer $\theta_{1}$ indifferent between buying good 1 with network $\theta_{2}-\theta_{1}$ or not consuming at all, and the upgrade price $p_{u}=\theta_{2} q_{\Delta}+\alpha\left[f\left(\theta_{2}, 1-\theta_{1}\right)-f\left(\theta_{2}, \theta_{2}-\theta_{1}\right)\right]$ makes consumer $\theta_{2}$ indifferent between just buying good 1 or "upgrading" to good 2 .

This will lead us to analyze profit functions of the form

$$
\left(1-\theta_{1}\right)\left[p_{1}\left(\theta_{1}, 1-\theta_{1}\right)-c_{1}\right]+\left(1-\theta_{2}\right)\left[p_{u}\left(\theta_{2}, 1-\theta_{2}\right)-c_{\Delta}\right]-\left(1-\theta_{1}\right) L\left(\alpha, \theta_{1}, \theta_{2}\right),
$$

where the first part of this expression is what profits in the good-1 market would be if good- 1 were purchased by all consumers with types at least $\theta_{1}$, the second term represents additional profits from sales of good 2, and the third term, $-\left(1-\theta_{1}\right) L\left(\alpha, \theta_{1}, \theta_{2}\right)$, captures the loss in revenue due to the reduction in consumer $\theta_{1}$ 's value of good 1 caused by sales of the incompatible good 2. (Because good 2 is backward compatible, there is not a corresponding term reflecting lost profits in market 2.) The focus of our analysis will be on how the specification of preferences determines the nature of this revenue loss, and on how the monopolist's loss of revenues compares to the loss of welfare suffered by consumers of good $1 .{ }^{14}$

${ }^{13}$ It is also possible that in some cases there is a negative correlation between the value of the basic good and the value of the network externality, although we think this is less likely. Such cases can be analyzed with the approach used here, provided that $\partial^{2} f / \partial x \partial \theta$ is not so negative that low $\theta$ consumers have a higher willingness to pay than consumers with higher $\theta$ 's. Note that the additive case is not a knife-edge here and should be a reasonable approximation of the outcome with a small amount of positive or negative correlation.

${ }^{14}$ This externality term is what most sharply differentiates this article from those on durable goods without compatibility issues.

(C) RAND 2000 
With additive preferences, the value of the network is the same for all consumers. We thus expect the monopolist to be able to capture all of the surplus attributable to the network, so that the monopolist's loss function $L$ should coincide with the loss in welfare; this will imply that the monopolist will never sell too many upgrades for a given level of supply of the "base" good. In contrast, with multiplicative preferences, the monopolist does not capture all of the benefits that the network provides to consumers, and so the monopolist can sell more of the upgrade than is optimal even given its sales of the base good. As we will see, in either case the monopolist's reduced supply of good 1 can lead it to provide more of good 2 than is optimal, and welfare can sometimes be improved by banning the sale of good 2 .

In addition to the externalities that good 2 imposes on consumers of good 1, there is an additional complication here that does not arise in standard durable-good models: Because of the "coordination-game" aspect of network effects, it is now possible that a given pair of prices $p_{1}, p_{u}$ can be consistent with several allocations $\theta_{1}, \theta_{2}$. Furthermore, since consumers are heterogeneous, they may not all have the same ordering of the possible equilibria, so we cannot use a Pareto criterion to select between the equilibria. Instead, we will make assumptions whose thrust is that network effects are not too large compared to other factors, and verify that under our assumptions the monopolist's chosen prices lead to a unique equilibrium allocation, so that we will not need to worry about multiple equilibria in the coordination games played by consumers.

As in the previous section, let $q_{\Delta}=q_{2}-q_{1}$ denote the difference in quality levels; let $c_{\Delta}=c_{2}-c_{1}$ be the difference in production cost. We assume throughout that $q_{1}>c_{1}>0$ and $q_{\Delta}>c_{\Delta}>0$.

$\square \quad$ Multiplicative preferences. In this section we consider the case where consumers have multiplicative preferences: consumer $\theta$ 's utility from a good of quality $q$ with network of size $x$ is $\theta q+\alpha \theta x$. We show not only that the existence of an upgrade can reduce welfare, but also that upgrades can be "excessive" in the sense that welfare can be improved if the monopolist sells fewer copies of the upgrade holding fixed sales of the base good. This type of excessive progress contrasts with what would be expected from thinking about the upgrade as a separate monopoly good, and it is also different from the usual distortion in metering models of price discrimination.

We will assume that network effects are "small" in the following sense:

Assumption 1. $q_{1}>2 \alpha, q_{1}-c_{1}>\alpha, q_{\Delta}>\alpha$, and $q_{\Delta}-c_{\Delta}>\alpha / 4$.

With multiplicative preferences, the marginal consumer of good 1 is willing to pay price

$$
p_{1}\left(\theta_{1}, \theta_{2}\right)=\theta_{1} q_{1}+\alpha \theta_{1}\left(\theta_{2}-\theta_{1}\right)
$$

and the marginal consumer of good 2 is willing to pay a premium of

$$
p_{u}\left(\theta_{1}, \theta_{2}\right)=\theta_{2} q_{\Delta}+\alpha \theta_{2}\left(1-\theta_{2}\right)
$$

for the upgrade from good 1 to good 2.

Our first task is to verify that the prices $p_{1}, p_{u}$ lead to a unique allocation.

Lemma 1. Fix an allocation $\theta_{1}, \theta_{2}$ with $0 \leq \theta_{1} \leq \theta_{2} \leq 1$, and let prices $p_{1}, p_{u}$ be given by (1) and (2). Under Assumption 1, $\theta_{1}, \theta_{2}$ is the unique allocation consistent with $p_{1}, p_{u}$. Moreover, $\theta_{1}, \theta_{2}$ satisfies 


$$
\begin{aligned}
& \theta_{2}=\min \left(1, \frac{\alpha+q_{\Delta}-\left[\left(\alpha+q_{\Delta}\right)^{2}-4 \alpha p_{u}\right]^{1 / 2}}{2 \alpha}\right) \text { and } \\
& \theta_{1}=\min \left(1, \frac{\alpha \theta_{2}+q_{1}-\left[\left(\alpha \theta_{2}+q_{1}\right)^{2}-4 \alpha p_{1}\right]^{1 / 2}}{2 \alpha}\right)
\end{aligned}
$$

Proof. See the Appendix.

Lemma 1 does not rule out the possibility that the monopolist might prefer to use prices other than those given by (1) and (2) to implement an allocation with $\theta_{1}=0$ or $\theta_{2}=1$, but it is easy to see that the restriction to (1) and (2) is without loss of generality: the monopolist cannot gain by setting a higher price than $p_{u}$ when sales of the upgrade are 0 , and $p_{1}=0$ is the profit-maximizing price to charge when implementing $\theta_{1}=0$. Similarly, the $p_{1}, p_{u}$ given by (1) and (2) is only one of the possible ways to implement an allocation with $\theta_{1}=\theta_{2}$ so that there are sales of only good 2, but all of these pricing schemes yield the same revenue.

The monopolist's profit function is

$$
\begin{aligned}
\pi\left(\theta_{1}, \theta_{2}\right)= & \left(1-\theta_{1}\right)\left[\theta_{1} q_{1}+\alpha \theta_{1}\left(1-\theta_{1}\right)-c_{1}\right]+\left(1-\theta_{2}\right)\left[\theta_{2} q_{\Delta}+\alpha \theta_{2}\left(1-\theta_{2}\right)-c_{\Delta}\right] \\
& -\alpha\left(1-\theta_{2}\right) \theta_{1}\left(1-\theta_{1}\right) .
\end{aligned}
$$

Lemma 2. Under Assumption 1, if the monopoly outcome $\theta_{1}^{m}, \theta_{2}^{m}$ has positive sales of both goods, it is a solution of the system

$$
\theta_{1}=\frac{q_{1}+\alpha\left(1+\theta_{2}\right)-\left[\left(q_{1}+\alpha\left(1+\theta_{2}\right)\right)^{2}-3 \alpha\left(q_{1}+\alpha \theta_{2}+c_{1}+\alpha \theta_{1}\left(1-\theta_{1}\right)\right]^{1 / 2}\right.}{3 \alpha}
$$

and

$$
\theta_{2}=\frac{q_{\Delta}+2 \alpha-\left[\left(q_{\Delta}+2 \alpha\right)^{2}-3 \alpha\left(q_{\Delta}+\alpha+c_{\Delta}+\alpha \theta_{1}\left(1-\theta_{1}\right)\right]^{1 / 2}\right.}{3 \alpha} .
$$

\section{Proof. See the Appendix.}

Lemma 2 only describes the outcome when the monopolist chooses to sell both goods. It is certainly possible that the monopolist will choose to sell only one of the two goods: if $q_{\Delta}$ is very small relative to $c_{\Delta}$, the monopolist will be tempted to sell only good 1 , and if $q_{\Delta}$ is sufficiently large, the monopolist will want to sell the upgrade to everyone. For a range of parameter values, however, the monopoly outcome will be interior and will be the solution to the first-order conditions. One example where this will occur is when $q_{\Delta} / c_{\Delta}<q_{1} / c_{1}$ and $\alpha$ small, because the solution converges to that given by Fudenberg and Tirole (1998) for the model without network effects. A second example is when $c_{1}=\alpha / 4$. In this case $\theta_{1}^{m}=1 / 2$ for any values of the other parameters, and $\left(\partial \pi / \partial \theta_{2}\right)(1 / 2,1 / 2)=c_{\Delta}>0$ implies that $\theta_{2}^{m}>1 / 2$.

Because the old good is not forward compatible with the new one, each unit of good 2 that is sold imposes a negative externality on users of good 1 . The term $\alpha \theta_{1}\left(1-\theta_{1}\right)$ in the first-order condition (6) for the choice of $\theta_{2}$ reflects the fact that the monopolist internalizes the loss to the marginal buyer of good 1 from marginal increases in the sales of good 2. This marginal private loss is less than the marginal social loss, which is $\alpha \int_{\theta_{1}}^{1} \theta d \theta$. If this effect is strong enough to outweigh the standard monopoly tendency to sell too little of any good, the monopolist will end up selling too many upgrades (holding fixed the level of $\theta_{1}$ ). 
To see that this does in fact occur at times in our model, we explicitly calculate social welfare when the monopolist chooses cutoffs $\theta_{1}$ and $\theta_{2}$. Social welfare is given by

$$
\begin{aligned}
W= & \int_{\theta_{1}}^{1} q_{1} \theta d \theta+\int_{\theta_{2}}^{1} q_{\Delta} \theta d \theta+\alpha\left(\theta_{2}-\theta_{1}\right) \int_{\theta_{1}}^{\theta_{2}} \theta d \theta+\alpha\left(1-\theta_{1}\right) \int_{\theta_{2}}^{1} \theta d \theta \\
& -c_{1}\left(1-\theta_{1}\right)-c_{\Delta}\left(1-\theta_{2}\right) \\
= & \left(\int_{\theta_{1}}^{1} q_{1} d \theta+\alpha\left(1-\theta_{1}\right) \int_{\theta_{1}}^{1} \theta d \theta-c_{1}\left(1-\theta_{1}\right)\right) \\
& +\left(\int_{\theta_{2}}^{1} q_{\Delta} d \theta+\alpha\left(1-\theta_{2}\right) \int_{\theta_{2}}^{1} \theta d \theta-c_{\Delta}\left(1-\theta_{2}\right)\right)-\alpha\left(1-\theta_{2}\right) \int_{\theta_{1}}^{1} \theta d \theta .
\end{aligned}
$$

Because the partial derivative of profits with respect to $\theta_{2}$ is zero at the monopoly outcome, it is helpful to consider the consumer surplus $C S=W-\pi$, which we will write as $C S_{1}+C S_{2}$, where

$$
\begin{aligned}
C S_{1}\left(\theta_{1}, \theta_{2}\right) & =\int_{\theta_{1}}^{1} q_{1} \theta d \theta+\alpha\left(1-\theta_{1}\right) \int_{\theta_{1}}^{\theta_{2}} \theta d \theta-\left(1-\theta_{1}\right) \theta_{1}\left(q_{1}+\alpha\left(\theta_{2}-\theta_{1}\right)\right) \\
& =\int_{\theta_{1}}^{1}\left(\theta-\theta_{1}\right)\left(q_{1}+\alpha\left(\theta_{2}-\theta_{1}\right)\right) d \theta=\frac{q_{1}\left(1-\theta_{1}\right)^{2}}{2}+\frac{\alpha\left(\theta_{2}-\theta_{1}\right)^{3}}{2}
\end{aligned}
$$

and

$$
C S_{2}\left(\theta_{1}, \theta_{2}\right)=\int_{\theta_{2}}^{1}\left(\theta-\theta_{2}\right)\left(q_{\Delta}+\alpha\left(1-\theta_{2}\right)\right) d \theta=\frac{q_{\Delta}\left(1-\theta_{2}\right)^{2}}{2}+\frac{\alpha\left(1-\theta_{2}\right)^{3}}{2} .
$$

Differentiating the expressions for consumer surplus, we obtain the following result.

Proposition 4. Holding fixed the level $1-\theta_{1}^{*}$ of sales of good 1, social welfare is improved by a marginal reduction in sales of good 2 if

$$
\frac{\alpha\left(1-\theta_{1}^{*}\right)^{2}}{2}>\frac{3 \alpha\left(1-\theta_{2}^{*}\right)^{2}}{2}+\left(1-\theta_{2}^{*}\right) q_{\Delta} ;
$$

welfare is harmed if the reverse inequality holds.

Proposition 4 shows that the monopolist sells too many upgrades given the level of the base good whenever the monopoly solution involves few sales of the upgrade. To verify that this situation is consistent with our other assumptions, consider the case $c_{1}=\alpha / 4$, where $\theta_{1}^{m}=1 / 2$ and $\theta_{2}^{m}>1 / 2$ is given by equation (6). Here

$$
\lim _{c_{\Delta} \rightarrow q_{\Delta}-(\alpha / 4)} \theta_{2}^{m}=1,
$$

so by choosing $c_{\Delta}$ close to $q_{\Delta}-\alpha / 4$ we can send the right-hand side of (7) to zero while the left-hand side remains fixed at $\alpha / 8$. 
Social welfare increases with small increases in sales of the upgrade if the reverse of (7) holds. Note that this must be the case in the limit as $\alpha \rightarrow 0$ : with negligible network effects the monopolist always sells too little of good 2. Here the externalities between users are unimportant, and the standard intuition about monopolies applies.

Because welfare can be increased by reducing sales of the upgrade holding sales of good 1 fixed, it is not very surprising that welfare can be increased by banning the upgrade altogether. For an example where this is true, consider again the case $c_{1}=\alpha / 4$. If the upgrade is banned, the monopolist will again choose $\theta_{1}^{m}=1 / 2$. Thus we can calculate

$$
W\left(\frac{1}{2}, 1\right)-W\left(\frac{1}{2}, \theta_{2}^{m}\right)=\left(1-\theta_{2}^{m}\right)\left[\alpha\left(\frac{\left(\theta_{2}^{m}\right)^{2}}{2}-\frac{1}{8}\right)-\left(q_{\Delta}\left(\frac{1+\theta_{2}^{m}}{2}\right)-c_{\Delta}\right)\right] .
$$

The term $\alpha\left[\left(\theta_{2}^{m 2}\right) / 2-1 / 8\right]$ corresponds to the increased network benefits to types between $1 / 2$ and $\theta_{2}^{m}$ when the upgrade is banned, and $q_{\Delta}\left[\left(1+\theta_{2}^{m}\right) / 2\right]-c_{\Delta}$ corresponds to the loss to those who had purchased the upgrade. As $c_{\Delta}$ approaches $q_{\Delta}-(\alpha / 4)$ from below, the first term converges to $3 \alpha / 8$ and the second converges to $q_{\Delta}-c_{\Delta}=\alpha / 4$, so welfare is increased by banning good 2 .

Proposition 5. Welfare can be increased by banning the sale of good 2 .

We have also investigated this question numerically; details are in the working paper version of this article, Ellison and Fudenberg (1999).

Additive preferences. With additive preferences, type $\theta$ assigns utility $u=\theta q+\alpha x$ to a good of quality $q$, where $x$ is the size of the associated network. Hence the marginal consumer of good 1 is willing to pay price

$$
p_{1}\left(\theta_{1}, \theta_{2}\right)=\theta_{1} q_{1}+\alpha\left(\theta_{2}-\theta_{1}\right)
$$

and the marginal consumer of good 2 will pay a premium of

$$
p_{u}\left(\theta_{1}, \theta_{2}\right)=\theta_{2} q_{\Delta}+\alpha\left(1-\theta_{2}\right)
$$

for the upgrade from good 1 to good 2.

As above, before proceeding to the analysis of the monopolist's maximization problem, we need to verify that prices $p_{1}, p_{u}$ lead to a unique allocation. To do so we again assume that network effects are comparatively small.

Assumption 2. $q_{1}>3 \alpha, q_{\Delta}>3 \alpha, q_{1}>c_{1}+\alpha$, and $q_{\Delta}>c_{\Delta}+\alpha$.

Lemma 3. Fix an allocation $\theta_{1}, \theta_{2}$ with $0 \leq \theta_{1} \leq \theta_{2} \leq 1$, and let prices $p_{1}, p_{u}$ be given by (9) and (10). Under Assumption 2, $\theta_{1}, \theta_{2}$ is the unique allocation consistent with prices $p_{1}, p_{u}$.

Proof. See the Appendix.

As in the multiplicative section, there are prices other than those given by (9) and (10) that can implement corner allocations, but none of them give higher revenue. Thus there is no loss of generality in writing the monopolist's profit function as 


$$
\begin{aligned}
\pi\left(\theta_{1}, \theta_{2}\right)= & \left(1-\theta_{1}\right)\left[\theta_{1}\left(q_{1}-\alpha\right)-\left(c_{1}-\alpha\right)\right]+\left(1-\theta_{2}\right)\left[\theta_{2}\left(q_{\Delta}-\alpha\right)-\left(c_{\Delta}-\alpha\right)\right] \\
& -\alpha\left(1-\theta_{1}\right)\left(1-\theta_{2}\right) .
\end{aligned}
$$

The last line of the expression above has three parts. The first is what profits in the good-1 market would be if good 2 were absent. The second term represents profits from sales of good 2 . The third term, $-\alpha\left(1-\theta_{1}\right)\left(1-\theta_{2}\right)$, is the reduction in the value of good 1 caused by sales of the incompatible good 2 .

At an interior solution, which means here both that each cutoff $\theta_{i} \in[0,1]$ and that $\theta_{1}<\theta_{2}$, the first-order conditions for maximizing profit yield

$$
\theta_{1}^{m}\left(\theta_{2}\right)=\frac{1}{2}+\frac{c_{1}-\alpha+\alpha\left(1-\theta_{2}\right)}{2\left(q_{1}-\alpha\right)}, \quad \theta_{2}^{m}\left(\theta_{1}\right)=\frac{1}{2}+\frac{c_{\Delta}-\alpha+\alpha\left(1-\theta_{1}\right)}{2\left(q_{\Delta}-\alpha\right)} .
$$

The following result is obtained by solving the above system and providing a condition under which the solution is interior.

Proposition 6. If $q_{\Delta}\left(c_{1}-\alpha\right)+\left(q_{\Delta}-\alpha\right) c_{1}<q_{1}\left(c_{\Delta}-\alpha\right)+\left(q_{1}-\alpha\right) c_{\Delta}$ and Assumption 2 is satisfied, the monopolist chooses to sell both the old good and the upgrade. The monopolist's optimal allocation is given by

$$
\begin{aligned}
& \theta_{1}^{m}=1-\frac{2\left(q_{\Delta}-\alpha\right)\left(q_{1}-c_{1}\right)-\alpha\left(q_{\Delta}-c_{\Delta}\right)}{4\left(q_{\Delta}-\alpha\right)\left(q_{1}-\alpha\right)-\alpha^{2}} \\
& \theta_{2}^{m}=1-\frac{2\left(q_{1}-\alpha\right)\left(q_{\Delta}-c_{\Delta}\right)-\alpha\left(q_{1}-c_{1}\right)}{4\left(q_{\Delta}-\alpha\right)\left(q_{1}-\alpha\right)-\alpha^{2}} .
\end{aligned}
$$

Proof. The second-order conditions are satisfied because it is sufficient that $q_{1}>\alpha$, $\Delta q>\alpha$. The assumptions that $q_{1}>3 \alpha, q_{\Delta}>3 \alpha$, and $q_{1}>c_{1}+\alpha, q_{\Delta}>c_{\Delta}+\alpha$ imply that both first-order equations have solutions between zero and one. Finally, straightforward but tedious calculation shows that the solution to the first-order conditions satisfies $\theta_{1}^{m}<\theta_{2}^{m}$ exactly when

$$
q_{\Delta}\left(c_{1}-\alpha\right)+\left(q_{\Delta}-\alpha\right) c_{1}<q_{1}\left(c_{\Delta}-\alpha\right)+\left(q_{1}-\alpha\right) c_{\Delta} .
$$

Remarks. (i) In the limit $\alpha \rightarrow 0$ of vanishing network effects, the condition $q_{\Delta}\left(c_{1}-\alpha\right)+\left(q_{\Delta}-\alpha\right) c_{1}<q_{1}\left(c_{\Delta}-\alpha\right)+\left(q_{1}-\alpha\right) c_{\Delta}$ simplifies to the condition $q_{\Delta} / c_{\Delta}<q_{1} / c_{1}$ found by Fudenberg and Tirole (1998). In particular, when good 1 and good 2 are equally costly (so that $c_{\Delta}=0$ ) and there are no network effects, the monopolist sells only good 2 .

(ii) The first-order conditions show that as $\theta_{2}$ falls, $\theta_{1}$ goes up. This is because in the additive model the upper bound on the extent of good 1's network $\left(1-\theta_{2}\right)$ enters in the same way as do costs, so a decrease in $\theta_{2}$ is like an increase in costs, which decreases sales. By definition, $\theta_{2}=1$ if only good 1 is available, so here the presence of the upgrade decreases sales of the base good.

Let us consider the monopolist's choice of $\theta_{2}$ in more detail. This maximization has two parts, profits in market 2 and externality on market 1 . As usual, maximizing profits in market 2 leads to undersupply of good 2. The externality term is $-\alpha\left(1-\theta_{1}\right)\left(1-\theta_{2}\right)$; because the network effect enters additively the externality is 
completely reflected in the monopolist's profit, so this is actually the social externality at the same level of $\theta_{1}$. This suggests that the monopolist is "internalizing the externality" caused by upgrades; to check that intuition we now look at the socially optimal allocation.

Social welfare is given by

$$
\begin{aligned}
W= & \int_{\theta_{1}}^{1} q_{1} \theta d \theta+\int_{\theta_{2}}^{1} q_{\Delta} \theta d \theta+\alpha\left(\theta_{2}-\theta_{1}\right)^{2}+\alpha\left(1-\theta_{2}\right)\left(1-\theta_{1}\right)-c_{1}\left(1-\theta_{1}\right)-c_{\Delta}\left(1-\theta_{2}\right) \\
= & \left(\int_{\theta_{1}}^{1} q_{1} \theta d \theta+\alpha\left(1-\theta_{1}\right)^{2}-c_{1}\left(1-\theta_{1}\right)\right)+\left(\int_{\theta_{2}}^{1} q_{\Delta} \theta d \theta+\alpha\left(1-\theta_{2}\right)^{2}-c_{\Delta}\left(1-\theta_{2}\right)\right) \\
& -\alpha\left(1-\theta_{1}\right)\left(1-\theta_{2}\right) .
\end{aligned}
$$

The expression in the first parenthesis is the welfare if only good 1 were sold, the second expression is the welfare from the upgrade if the upgrade were fully compatible, and the third term is the externality that the upgrade market has on good 1 .

Consumer surplus can be written as the difference between welfare and profits

$$
\begin{aligned}
\operatorname{CS}\left(\theta_{1}, \theta_{2}\right)= & \left(\int_{\theta_{1}}^{1} q_{1} \theta d \theta+\alpha\left(1-\theta_{1}\right)^{2}-c_{1}\left(1-\theta_{1}\right)\right) \\
& +\left(\int_{\theta_{2}}^{1} q_{\Delta} \theta d \theta+\alpha\left(1-\theta_{2}\right)^{2}-c_{\Delta}\left(1-\theta_{2}\right)\right)-\alpha\left(1-\theta_{1}\right)\left(1-\theta_{2}\right) \\
& -\left(1-\theta_{1}\right)\left[\theta_{1}(q-\alpha)-\left(c_{1}-\alpha\right)\right]-\left(1-\theta_{2}\right)\left[\theta_{2}\left(q_{\Delta}+\alpha\right)-\left(c_{\Delta}-\alpha\right)\right] \\
& +\alpha\left(1-\theta_{1}\right)\left(1-\theta_{2}\right) \\
= & \left(\int_{\theta_{1}}^{1} q_{1}\left(\theta-\theta_{1}\right) d \theta\right)+\left(\int_{\theta_{2}}^{1} q_{\Delta}\left(\theta-\theta_{2}\right) d \theta\right) .
\end{aligned}
$$

Note that there is no interaction between $\theta_{1}$ and $\theta_{2}$ in this expression: Because the monopolist internalizes the externality caused by incompatibility, it cancels out in the subtraction. For any fixed $\theta_{1}$, the fact that $\left(\partial \pi / \partial \theta_{2}\right)\left[\theta_{1}, \theta_{2}^{m}\left(\theta_{1}\right)\right]=0$ implies that

$$
\frac{\partial W}{\partial \theta_{2}}\left[\theta_{1}, \theta_{2}^{m}\left(\theta_{1}\right)\right]=\frac{\partial C S}{\partial \theta_{2}}\left[\theta_{1}, \theta_{2}^{m}\left(\theta_{1}\right)\right]<0 .
$$

Hence, in contrast to the multiplicative case, sales of the upgrade can never be excessive given $\theta_{1}$.

While upgrades are never excessive in the sense of being too high conditional on the level of sales of good 1, it is possible that the monopolist may choose to sell more units of the upgrade than would a social planner, because the monopolist's reduced sales of good 1 reduce the negative externality from selling good 2 .

Proposition 7. (i) Given Assumption 2, profit-maximizing sales of good 2 for fixed sales of good 1 are not higher than the social optimum. They are strictly lower provided (c) RAND 2000 . 
that the constraint that sales of the upgrade can be no larger than sales of good 1 does not bind.

(ii) The monopoly outcome can have more sales of good 2 than the social optimum.

Proof. Given Assumption 2, $W$ and $\pi$ are both concave in $\theta_{2}$ for any $\theta_{1}$. If $\theta_{2}^{m}\left(\theta_{1}\right)>\theta_{1}$, the monopoly outcome is given by the first-order conditions and (i) follows from the discussion above. Otherwise, $W$ and $\pi$ are both decreasing in $\theta_{2}$ for $\theta_{2}>\theta_{1}$, and setting $\theta_{2}=\theta_{1}$ is both profit maximizing and socially optimal. For (ii) note that if $c_{1}=0$, $q_{\Delta}=c_{\Delta}+\alpha, q_{1}>2 \alpha$, and $c_{\Delta}>\alpha / 2$, then maximizing the social welfare function gives that the social optimum is $\theta_{1}=0, \theta_{2}=1$. Profits are concave in each of $\theta_{1}$ and $\theta_{2}$ holding the other fixed, and the solutions to the monopoly first-order conditions are always interior. Hence, the monopoly solution is given by the first-order condition and, in particular $\theta_{2}^{m}=\theta_{2}^{m}\left(\theta_{1}^{m}\right)=1-\left(\alpha \theta_{1}^{m} / 2 c_{\Delta}\right)<1$. Q.E.D.

The proposition above illustrates an implication of the fact that $d \theta_{2}^{m} / d \theta_{1}^{m}<0$, namely that the monopolist's inefficiently low production of good 1 may lead to more production of good 2 than in the social optimum. The fact that $d \theta_{1}^{m} / d \theta_{2}^{m}<0$ also has an interesting efficiency consequence.

Proposition 8. Welfare can be increased by banning the sale of good 2 .

Intuitively, the reason why this is possible is that constraining the monopolist to increase $\theta_{2}$ will lead him to decrease $\theta_{1}$. From the formula for consumer surplus above,

$$
\frac{\partial C S}{\partial \theta_{1}}=-q_{1}\left(1-\theta_{1}\right) \quad \text { and } \quad \frac{\partial C S}{\partial \theta_{2}}=-q_{\Delta}\left(1-\theta_{2}\right)
$$

Thus, the welfare effect of banning good 2 is clearest if the unconstrained monopoly solution has $\theta_{2}^{m}$ close to one-the social loss from increasing $\theta_{2}$ is small and the gain from the resulting decrease in $\theta_{1}$ is larger, while constraining $\theta_{2}$ to be slightly higher than $\theta_{2}^{m}$ has no first-order effect on profits. Beyond this limit case, Ellison and Fudenberg (1999) exhibits a range of values of $q_{1}$ and $q_{\Delta}$ for which the welfare effect is positive. The key factor seems to be the magnitude of $q_{\Delta}$ : when it is large, the direct effect of the improvement outweighs the induced reduction in sales of the base good.

\section{Conclusion}

- We have discussed two reasons that a monopolist might supply upgrades in cases when it would be socially optimal to withhold them. Of the many factors not in the model that could have an important influence on our findings, the role of actual and potential competition is perhaps the most notable. In particular, the monopolist might have an additional incentive to introduce feature-packed upgrades to avoid leaving a market niche open for a potential entrant. At the same time, we should make clear that while we think the article's welfare comparisons are informative, they should not be read as a call for immediate government intervention.

\section{Appendix}

\footnotetext{
- Proofs of Lemmas 1-3 follow.
}

Proof of Lemma 1. Consider first equation (2), which is a quadratic in $\theta_{2}$ with a negative coefficient on the quadratic term. Let the right-hand side of (2) define the function $g\left(\theta_{2}\right)$; then $g(0)=0$ and $g(1)=q_{\Delta}$. Since $g^{\prime}(1)=q_{\Delta}-\alpha$, Assumption 1 implies that $g^{\prime}(1)>0$, so there is at most one $\theta_{2} \in[0,1]$ where the righthand side of (2) equals a given $p_{u}$. Moreover, since $g(1)=q_{\Delta}$ and $g$ is increasing, if $p_{u}$ is consistent with a 
$\theta_{2}<1$, then $p_{u}<q_{\Delta}$, so there is no equilibrium where $\theta_{2}=1$. Hence, $p_{u}$ leads to a unique $\theta_{2}$. Holding this value of $\theta_{2}$ fixed, a similar argument shows that there is a unique $\theta_{1}$ that solves (1). Q.E.D.

Proof of Lemma 2. If the monopoly outcome has nonzero sales of both goods, it must satisfy the first-order conditions

$$
\begin{aligned}
& \frac{\partial \pi}{\partial \theta_{1}}=\left(1-2 \theta_{1}\right)\left(q_{1}+\alpha \theta_{2}\right)-\alpha\left(2 \theta_{1}-3 \theta_{1}^{2}\right)+c_{1}=0, \\
& \frac{\partial \pi}{\partial \theta_{2}}=\left(1-2 \theta_{2}\right)\left(q_{\Delta}+\alpha\right)-\alpha\left(2 \theta_{2}-3 \theta_{2}^{2}\right)+c_{\Delta}+\alpha \theta_{1}\left(1-\theta_{1}\right)=0,
\end{aligned}
$$

along with the constraints $0 \leq \theta_{1}<\theta_{2}<1$.

The first-order condition (A1) is a quadratic, and so has two solutions. Profits are cubic in $\theta_{1}$ with a positive coefficient on the third-order term, and from Assumption 1

$$
\left.\frac{\partial \pi}{\partial \theta_{1}}\right|_{\left(0, \theta_{2}\right)}=q_{1}+\alpha \theta_{2}+c_{1}>0, \quad \text { and }\left.\quad \frac{\partial \pi}{\partial \theta_{1}}\right|_{\left(1, \theta_{2}\right)}=-q_{1}-\alpha \theta_{2}+\alpha+c_{1}<0
$$

Therefore both roots are real; denote them $\theta_{1}^{L}, \theta_{1}^{H}$ with $0<\theta_{1}^{L}<1<\theta_{1}^{H}$. Hence the profit-maximizing value of $\theta_{1}$ in the range $\left[0, \theta_{2}\right]$ is $\theta_{1}^{L}$ if $\theta_{1}^{L}<\theta_{2}$ and $\theta_{2}$ otherwise, where $\theta_{1}^{L}$ is given by (5).

Similarly, profits are cubic in $\theta_{2}$ with a positive coefficient on the third-order term, and under Assumption 1 we have

$$
\left.\frac{\partial \pi}{\partial \theta_{2}}\right|_{\left(\theta_{1}, 0\right)}=q_{\Delta}+\alpha+c_{\Delta}+\alpha \theta_{1}\left(1-\theta_{1}\right)>0 \quad \text { and }\left.\quad \frac{\partial \pi}{\partial \theta_{2}}\right|_{\left(\theta_{1}, 1\right)}=-q_{\Delta}+c_{\Delta}+\alpha \theta_{1}\left(1-\theta_{1}\right)<0,
$$

so as above the smaller root of the first-order condition (A2) is a local maximum and lies strictly between zero and one. Hence the profit-maximizing value of $\theta_{2}$ in the range $\left[\theta_{1}, 1\right]$ is $\theta_{2}^{L}$ if $\theta_{2}^{L}>\theta_{1}$ and $\theta_{1}$ otherwise, where $\theta_{2}^{L}$ is given by (6). Q.E.D.

Proof of Lemma 3. Note first that (9) and (10) are linear and invertible, with inverses $\theta_{1}=\left(p_{1}-\alpha \theta_{2}\right) /\left(q_{1}-\alpha\right)$ and $\theta_{2}=\left(p_{u}-\alpha\right) /\left(q_{\Delta}-\alpha\right)$. Thus any allocations other than $\theta_{1}, \theta_{2}$ that are consistent with $p_{1}, p_{u}$ must be corner allocations where there are no consumers on one or both of the margins. If $0<\theta_{2}<1$, then $\alpha<p_{u}\left(\theta_{1}, \theta_{2}\right)<q_{\Delta}$, so types near 1 must buy the upgrade and types near 0 will not. If $\theta_{2}=1$, then $p_{u}\left(\theta_{1}, \theta_{2}\right)=q_{\Delta}$, and because $q_{\Delta}>\alpha$, there is no interior equilibrium; if $\theta_{1}=\theta_{2}=0$, then $p_{u}=\alpha<q_{\Delta} / 3$ and $p_{1}=0$, so all types $\theta>1 / 3$ want to buy good 2 regardless of the actions of other consumers; iterating shows that all consumers must purchase both goods. If $\theta_{1}>0$, then $p_{1}\left(\theta_{1}, \theta_{2}\right)>\alpha \theta_{2}$, so there is no equilibrium where all consumers buy good 1 and some do not buy good 2, and to have all consumers buy good 2 would require $p_{1}+p_{u}=q_{1} \theta_{1}+q_{\Delta} \theta_{2}+\alpha\left(1-\theta_{2}\right) \leq \alpha$, which contradicts $q_{\Delta}>\alpha$. Finally, if $\theta_{1}=0<\theta_{2}$, then $p_{1}=\alpha \theta_{2}$ and all types above 0 want to buy good 1. Q.E.D.

\section{References}

Bensaid, B. AND Lesne, J.-P. “Dynamic Monopoly Pricing with Network Externalities.” International Journal of Industrial Organization, Vol. 14 (1996), pp. 837-855.

Bulow, J.I. "Durable-Goods Monopolists." Journal of Political Economy, Vol. 90 (1982), pp. 314-332.

COASE, R.H. "Durability and Monopoly." Journal of Law and Economics, Vol. 15 (1972), pp. 143-149.

CHOI, J.P. "Network Externality, Compatibility Choice, and Planned Obsolescence." Journal of Industrial Economics, Vol. 42 (1994), pp. 167-182.

DENECKERE, R.J. AND MCAFEe, R.P. “Damaged Goods." Journal of Economics and Management Strategy, Vol. 5 (1996), pp. 149-174.

Ellison, G. AND FudEnBERG, D. "The Neo-Luddite's Lament: Excessive Upgrades in the Software Industry." Harvard Institute for Economic Research Discussion Paper no. 1870, 1999.

FARrell, J. AND SALONER, G. "Standardization, Compatibility, and Innovation." RAND Journal of Economics, Vol. 16 (1985), pp. 70-83.

Fudenberg, D. AND Tirole, J. "Upgrades, Tradeins, and Buybacks.” RAND Journal of Economics, Vol. 29 (1998), pp. 235-258.

KAtZ, M.L. AND Shapiro, C. "Technology Adoption in the Presence of Network Externalities." Journal of Political Economy, Vol. 94 (1986), pp. 822-841.

(c) RAND 2000 . 
Mussa, M. And Rosen, S. "Monopoly and Product Quality.” Journal of Economic Theory, Vol. 18 (1978), pp. 301-317.

NAHM, J. "Endogenous Innovation with Coasian Dynamics: A Comment on Waldman (1996)." Mimeo, Department of Economics, Harvard University, 1998.

. "The Effects of the Compatibility Decision on Software and Hardware Markets." Mimeo, Department of Economics, Harvard University, 1999.

Spence, A.M. "Monopoly, Quality and Regulation." Bell Journal of Economics Vol. 6 (1975), pp. 417-429. . "Product Differentiation and Welfare." American Economic Review, Vol. 66 (1976), pp. 407-414.

Stokey, N.L. "Rational Expectations and Durable Good Pricing." Bell Journal of Economics, Vol. 12 (1981), pp. 112-128.

Waldman, M. “A New Perspective on Planned Obsolescence.” Quarterly Journal of Economics, Vol. 108 (1993), pp. 273-283.

. "Planned Obsolescence and the R\&D Decision." RAND Journal of Economics, Vol. 27 (1996), pp. 583-595. 